\title{
Exact Bit Error-Rate Analysis of Two-User NOMA Using QAM with Arbitrary Modulation Orders
}

\author{
T. Assaf, Member, IEEE, A. Al-Dweik, Senior Member, IEEE, M. El Moursi, Senior Member, IEEE, H. Zeineldin, \\ Senior Member, IEEE, and M. Al-Jarrah, Member, IEEE
}

\begin{abstract}
This work considers the exact bit error rate (BER) analysis of a two-user non-orthogonal multiple access (NOMA) system using quadrature amplitude modulation (QAM). Unlike existing work, no constraints on the modulation order of the QAM symbols for any user. Closed-form expressions are derived for the BER of joint multiuser detector (JMuD), which is demonstrated that it is equivalent to the successive interference cancellation (SIC) receiver. Moreover, a general expression is derived for the relation between the power allocation factors for the two users, which depends on the modulation order for each user.
\end{abstract}

Index Terms-Non-orthogonal multiple access, NOMA, bit error rate, BER, joint detection, successive interference cancellation, SIC, quadrature amplitude modulation, QAM.

\section{INTRODUCTION}

Non-orthogonal multiple access (NOMA) is an efficient multiple access technique, which is considered as a promising candidate for future wireless communication networks. NOMA can improve the spectral efficiency by allowing multiple users to share the transmission resources simultaneously, at the expense of some additional receiver complexity and bit error rate (BER) degradation [1]. Several NOMA schemes have been proposed in the literature, but the main categories are the code-domain [2], [3] and power-domain NOMA [4], [5], which is the focus of this work. In the literature, successive interference cancellation (SIC) has been widely considered as the main detection scheme for power-domain NOMA [3], [6]-[11]. However, SIC detectors (SICDs) suffer from long processing times because the $n$th user has to sequentially detect and subtract the signals of all users whose indices are less than $n$ [12]. To reduce the processing time, joint multiuser detection (JMuD) has been proposed as an alternative for the SICD [12]-[15].

Bit error rate (BER) analysis of NOMA using SICD has received extensive attention in the recent literature. For example, the authors of [11] derived the pairwise error probability for downlink NOMA using quadrature phase shift keying (QPSK), and used the union bound to represent the BER. However, the bound may considerably deviate from the actual BER in several operating conditions. In [6], the BER performance of two and three-user downlink NOMA systems with QPSK over Nakagami$m$ channels using SICD, and hence, the main drawback is that

T. Assaf, A. Al-Dweik, M. El Moursi and H, Zeineldin are with the Department of Electrical and Computer Engineering, Khalifa University, Abu Dhabi 127788, United Arab Emirates. (E-mail: \{tasneem.assaf, arafat.dweik, mohamed.elmoursi, hatem.zeineldin\}@ku.ac.ae).

M. Al-Jarrah is with the School of Electrical and Electronic Engineering, University of Manchester, Manchester M13 9PL, U.K. (e-mail: mohammad.aljarrah@manchester.ac.uk) derived expressions are not applicable for higher order quadrature amplitude modulation (QAM). In [16], the BER performance for three-user downlink NOMA network using SICD is investigated where space shift keying (SSK) is considered. The main limitation is that the BER expression is only valid for QPSK scheme. The BER is investigated for both downlink and uplink NOMA systems using SICD under Rayleigh fading channels in [8], where exact and approximate closed-form expressions are derived for downlink and uplink, respectively. The derived expressions are applicable only two-user NOMA systems using BPSK and QPSK. In [17], the BER for downlink NOMA systems is derived for any number of users using binary phase shift keying (BPSK) considering SICD. Exact symbol error rate analysis of downlink NOMA using QAM is presented in [18]. Although SER is a useful indicator for the system error performance, the BER is more informative and it is the standard metric for error rate performance.

Although the error rate analysis of NOMA systems has recently attracted extensive research, to the best of the authors' knowledge, there is no work in the literature which considers the exact BER analysis of NOMA systems using QAM with arbitrary modulation orders for the individual users. The main objective of this work is to derive the exact BER for two-user NOMA system which considers QAM with arbitrary modulation orders. JMuD is used as detection scheme for the NOMA system. Furthermore, a general expression is derived for the relation between the power allocation factors for the two users, which depends on the modulation order for each user. Moreover, we demonstrate that the SICD is just a low complexity implementation of the maximum likelihood detector (MLD), which is denoted as the $\mathrm{JMuD}$.

The rest of the paper is organized as follows. In Sec. II, the system and channel models are presented. The JMuD and SICD schemes are represented in Sec. III and IV, respectively. The exact BER analysis for the first and second users are presented in Sec. V and VI, respectively. The numerical and simulation results are shown in Sec. VII. Finally, the work is concluded in Sec. VIII.

\section{A. Notations}

To notations used throughout the paper are shown in Table I.

\section{System ModeL}

Consider a downlink power-domain NOMA system that supports two simultaneous users, $U_{1}$ and $U_{2}$. The users' equipment (UEs) and the base station (BS) are equipped with single antennas [6], and the data for the $n$th user is modulated using a square 


\begin{tabular}{|l|l|}
\hline$B_{n}=\log _{2} M_{n}$ & $v_{n}=\log _{2} \sqrt{M_{n}}$ \\
\hline$m=\max \left(M_{1}, M_{2}\right)$ & $M=\prod_{n=1}^{N} M_{n}$ \\
\hline$\Lambda_{n}=\sqrt{M_{n}}-1$ & $L_{1, n}=\left(1-2^{-i}\right) \Lambda_{n}$ \\
\hline$\varphi_{1}=\frac{1}{4}\left(\sqrt{\beta_{1}}+\sqrt{\beta_{2}}\right)$ & $L_{2, n}=\left\lceil\frac{2\left(\sqrt{M_{n}}-1\right)}{i}\right\rceil$ \\
\hline$\varphi_{2}=\frac{1}{4}\left(\sqrt{\beta_{1}}-\sqrt{\beta_{2}}\right)$ & $\ell_{i, n}=\left\lceil\frac{i-1}{\sqrt{M_{n}}}\right\rceil$ \\
\hline$\lambda_{i, j, n}=\left\lfloor\frac{j 2^{i-1}}{\sqrt{M_{n}}}\right\rfloor$ & $\rho_{i, j}=\left\lfloor\frac{j(i-1)}{v_{2}-1}\right\rfloor$ \\
\hline$g_{n}^{ \pm}(a, b)=\epsilon_{n}\left(a \sqrt{\frac{\beta_{1}}{E_{1}}} \pm b \sqrt{\frac{\beta_{2}}{E_{2}}}\right)$ & $\epsilon_{n}=\frac{\alpha_{n}}{\sigma_{n_{n}}}$ \\
\hline$Q\left(g_{1}(a, b)\right)=Q\left(g_{1}^{+}(a, b)\right)-Q\left(g_{1}^{-}(a, b)\right)$ \\
\hline
\end{tabular}

TABLE I

NOTATIONS

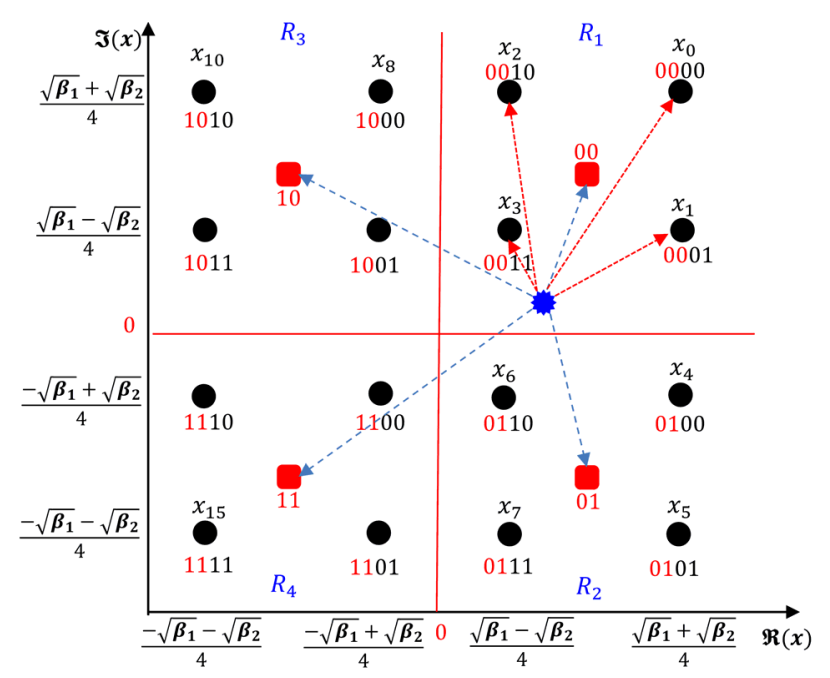

Fig. 1. The constellation diagram of the transmitted symbol $x$ for $N=2$.
QAM with modulation order $M_{n}$. Gray coding is used to map $B_{n}$ bits of each user before being multiplexed to form the NOMA symbol. The in-phase and quadrature components of a QAM symbol are respectively given by

$$
A_{n_{I}}= \pm\left(2 k_{n}-1\right), \quad k_{n} \in\left\{ \pm 1, \pm 3, \ldots, \pm \Lambda_{n}\right\}
$$

and

$$
A_{n_{q}}= \pm\left(2 l_{n}-1\right), l_{n} \in\left\{ \pm 1, \pm 3, \ldots, \pm\left(\Lambda_{n}\right)\right\} .
$$

Given that all symbols are equally probable with unity minimum Euclidean distance, the average energy for the $n$th user can be expressed as

$$
E_{n}=\frac{2}{3}\left(M_{n}-1\right) .
$$

The NOMA multiuser signal transmitted from the BS can be described as

$$
x=\sum_{n=1}^{N} \sqrt{\frac{\beta_{n} P_{T}}{E_{n}}} s_{n}
$$

where $s_{n}$ is the data symbol of the $n$th user that is selected uniformly from a gray coded QAM constellation with modulation order $M_{n}, P_{T}$ is the BS total transmit power. which is normalized to unity, and $\beta_{n}$ is the power allocation coefficient for the $n$th user, $\sum_{n=1}^{N} \beta_{n}=1$. Given that the transmitted symbol of the $n$th user is represented as $s_{n}=A_{n_{I}}+j A_{n_{q}}$, then $\mathbb{E}\left[|x|^{2}\right]=1$, where $\mathbb{E}[\cdot]$ denotes the statistical average. The symbol $s_{n} \in \mathbb{S}_{n}=\left\{d_{0}, d_{1}, \ldots, d_{M_{n}-1}\right\}$.

In flat fading channels, the received signal at the $n$th UE can be expressed as

$$
r_{n}=h_{n} x+w_{n}
$$

where $h_{n}$ represents the complex channel frequency response between the BS and the $n$th user, the channel gain $\left|h_{n}\right| \triangleq \alpha_{n}$, and $w_{n}$ is the additive white Gaussian noise (AWGN), $w_{n} \sim$ $\mathcal{C N}\left(0, N_{0}\right)$. In NOMA systems, it is typically assumed that $\alpha_{1}<\alpha_{2}$. Thus, the power coefficients should be allocated in the opposite order of the channel gains, i.e., $\beta_{1}>\beta_{2}$. Moreover, for proper implementation of NOMA systems and avoid overlap between the users' symbols, the power allocation coefficients must satisfy the following range constraint,

$$
\frac{\beta_{1}}{\beta_{2}}>\frac{M_{1}-1}{M_{2}-1} \Lambda_{2}^{2} \text {. }
$$

An example for the case of $N=2$ is shown in Fig. 1 where $M_{1}=M_{2}=4$. In the figure, the first user bits are the leftmost two bits and the second user bits are the rightmost two bits. As can be noted from the figure, the NOMA symbol $x$ has a 16-point constellation.

For coherent detection, the channel phase $\arg \left\{h_{n}\right\} \triangleq \theta_{n}$ should be estimated and compensated in the received signal. The received signal after phase equalization can be written as

$$
\check{r}_{n}=r_{n} \mathrm{e}^{-j \theta_{n}}=\alpha_{n} x+\check{w}_{n}
$$

where $\check{w}_{n}=w_{n} \mathrm{e}^{-j \theta_{n}}$. Given that $w_{n}$ is circularly symmetric, then $\check{w}_{n} \sim w_{n} \sim \mathcal{C N}\left(0, N_{0}\right)$. To extract the information symbols $s_{n} \forall n$, two possible detectors can be utilized, the SICD, and $\mathrm{JMuD}$ which can give identical error performance. The JMuD is similar to the MLD used with QAM signals, except that the bits in each symbol belong to multiple users.

\section{JMUD FOR NOMA SYMBOLS}

Based on the constellation diagram of a general NOMA system, e.g. Fig. 1 , the JMuD of the $n$th user can be written as,

$$
\left\{\hat{s}_{1}, \hat{s}_{2}, \ldots, \hat{s}_{N}\right\}=\arg \min _{s_{i} \in \mathbb{S}_{i}}\left|r_{n}-\hat{h}_{n} \sum_{i=1}^{N} \sqrt{\frac{\beta_{i}}{E_{i}}} s_{i}\right|^{2}
$$

where $\hat{h}_{n}$ is the estimated value of $h_{n}$. Therefore, the JMuD computes the Euclidean distance (ED) between the received signal and possible constellation pints, and selects the minimum ED. The number of constellation points in this case is $M$, and thus, the complexity of the detector increases exponentially as a function of the number of users. However, by noting the constellation in Fig. 1, it can be noted that the bits of $U_{1}$ in each of the four quadrants $\left\{R_{0}, R_{1}, R_{2}, R_{3}\right\}$ are fixed regardless the bits of $U_{2}$. Therefore, computing and comparing the EDs with any four symmetrical points would result in the same decision when the 
ED with the 16 constellation points is computed. For example, the constellation points that correspond to $x_{0}, x_{6}, x_{10}$ and $x_{15}$ in Fig. 1 are symmetrical and they can be used for detecting $\hat{s}$. Or equivalently, we can use $M_{1}$ virtual constellation points obtained from the scaled set $\sqrt{\frac{\beta_{i}}{E_{i}}} \mathbb{S}_{1}$, which are marked using the solid squares in Fig. 1. Consequently, the $\mathrm{JMuD}$ in (8) for $U_{1}$ is reduced to

$$
\hat{s}_{1}=\arg \min _{s_{1} \in \mathbb{S}_{1}}\left|r_{1}-\hat{h}_{1} \sqrt{\frac{\beta_{1}}{E_{1}}} s_{1}\right|^{2}
$$

which is the conventional MLD for a QAM signal. It is worth noting that the detector in (9) is identical to the SICD for $U_{1}$ [6]. Consequently, the JMuD and SICD are identical for the case of $U_{1}$ in terms of complexity and BER.

For the case of $U_{2}$ bits, the rightmost bits, it can be noted that the constellation points in each quadrant are identical. For example, the rightmost two bits in $\left\{x_{0}, x_{8}\right\}$ are equal, and the same note can be made for $\left\{x_{2}, x_{10}\right\}$ and $\left\{x_{1}, x_{5}\right\}$. Therefore, if the received signal is in quadrant $R_{i}$, then the point with the minimum ED will necessarily be one of the constellation points in $R_{i}$. Thus, given that we know the received signal falls in which quadrant, we limit the ED computation to the constellation points in that quadrant. However, the quadrant can be easily identified from the $U_{1}$ estimated symbol where $00 \rightarrow R_{0}, 01 \rightarrow R_{1}$, and so forth. Once the quadrant is identified, the detection process can be performed by shifting the quadrant center to the $\{0,0\}$ point, and then use conventional MLD of order $M_{2}$ to recover $\hat{s}_{2}$. By noting that the centers of the four quadrants are actually the virtual constellation points of $U_{1}$, it can be realized that the $\mathrm{JMuD}$ for $U_{2}$ is also equivalent to the SICD [6], except that the SICD has lower complexity. It is interesting to note that selecting the wring quadrant is equivalent to the unsuccessful SIC process.

\section{Detection of NOMA SignAls USING SICD}

The NOMA signals can also be detected using the SICD approach, where the signal for the $n$th user is detected after detecting and subtracting the signals of the first $n-1$ users. Therefore, MLD is applied $n$ times, however, the constellation size in each round is equal to the modulation order of the $n$th user signal, and thus,

$$
\hat{s}_{n}=\arg \min _{\tilde{s}_{n}}\left|r_{n}-\hat{h}_{n} \sum_{i=1}^{n-1} \sqrt{\frac{\beta_{i}}{E_{i}}} \hat{s}_{i}-\hat{h}_{n} \sqrt{\frac{\beta_{n}}{E_{n}}} \tilde{s}_{n}\right|^{2} .
$$

For the first user, it is clear that both the MLD (9) and SICD (10) have the same structure, and hence, the same BER, which is given in [6]. Moreover, it is worth noting the similarity between the SICD and JMuD in the sense that the signals of $U_{1}, \ldots, U_{n-1}$ are involved in the detection of the signal of $U_{n}$.

\section{BER ANALYSIS OF $U_{1}$}

The bit representation for each constellation point in the NOMA symbol can be expressed as $b_{n i},\{n, i\}$, where $n \in\{1,2\}$ is the user index, $i \in\left\{1,2, \ldots, B_{n}\right\}$ is the bit index. For the first user, the detection is performed using (9) where the first leftmost $B_{1}$ bits belong to the first user. To simplify the presentation, we initially consider the case of $M_{1}=M_{2}=4$ shown in Fig. 1, and then the approach is generalized for an arbitrary $M_{n}$. For this scenario, each NOMA symbol consists of 4 bits, 2 bits from each user.

For $b_{11}$, the constellation can be divided into two regions based on the decision boundary, which is the $y$-axis. In this case, the decision boundary and the constellation point can be at a distance of $\varphi_{1}$ or $\varphi_{2}$. The error event for $b_{11}$ depends on the transmitted NOMA symbol. For example, if $x_{0}, x_{1}, x_{4}$ or $x_{5}$ is transmitted, then $\operatorname{Pr}\left(\hat{b}_{11} \neq b_{11}\right)=\operatorname{Pr}\left(\mathfrak{n}_{1}<-\varphi_{1}\right)$, $\Re\left(\check{w}_{1}\right) \triangleq \mathfrak{n}_{1}$, and similarly, if $x_{2}, x_{3}, x_{6}$ or $x_{7}$ is transmitted, then $\operatorname{Pr}\left(\hat{b}_{11} \neq b_{11}\right)=\operatorname{Pr}\left(\mathfrak{n}_{1}<-\varphi_{2}\right)$. The case for $b_{12}$ is similar to $b_{11}$, except that the decision boundary in this case is the $x$-axis. Therefore, it is straightforward to show that $P_{b_{11}} \mid h_{1}=$ $P_{b_{12}} \mid h_{1}$. By considering all cases and taking into consideration that $P_{b_{11}}\left|h_{1}=P_{b_{12}}\right| h_{1}$, the average conditional BER for $U_{1}$ can be expressed as

$$
\begin{aligned}
P_{U_{1}} \mid h_{1} & =\frac{1}{2} \sum_{i=0}^{2} P_{b_{1 i}} \mid h_{1} \\
& =\frac{1}{2}\left[Q\left(\epsilon_{1} \varphi_{1}\right)+Q\left(\epsilon_{1} \varphi_{2}\right)\right]
\end{aligned}
$$

where $Q(\cdot)$ is the $Q$-function and $\sigma_{\mathfrak{n}_{1}}^{2}=N_{0} / 2$.

After exhaustive study to the NOMA constellation where every user uses QAM with arbitrary $M_{n}$, it can be noted that the errors events have specific patterns and features that allow extending the analysis for an arbitrary $M_{n}$. One of the main features is that the error probabilities of the $\frac{1}{2}\left(B_{1}+B_{2}\right)$ leftmost and rightmost are identical, and hence, only one of them should be derived. Therefore, the generalization of the same approach used in the example yields the following for an arbitrary $M_{n}$

$$
\begin{aligned}
P_{b_{1 i}} \mid h_{1} & =\frac{1}{\sqrt{M}} \sum \sum C\left(b_{1 i}\right) \\
\times & Q\left(\epsilon_{1}\left[F_{1}\left(b_{1 i}\right) \sqrt{\frac{\beta_{1}}{E_{1}}}+F_{2}\left(b_{1 i}\right) \sqrt{\frac{\beta_{2}}{E_{2}}}\right]\right) .
\end{aligned}
$$

For $b_{11}, C\left(b_{11}\right)=1$, and it is repeated $2 B_{2}$ times. For the $b_{1 i}$ where $i=v_{1}$, the value of the first term of $C\left(b_{1 v_{1}}\right)$ begins with $\sqrt{M_{1}} / 2$, which is repeated $\Lambda_{2}$ times. The $\left(\sqrt{M_{2}}+1\right)$ th value of $C\left(b_{1 v_{1}}\right)$ is the first value decremented by 1 and repeated $\Lambda_{2}$ times. The $\left(3 \sqrt{M_{2}}\right)$ th value is the $\left(\sqrt{M_{2}}+1\right)$ th term with a different sign and repeated $\Lambda_{2}$. This pattern repeats for the remaining terms until the value of becomes $C\left(b_{1 v_{1}}\right)$ becomes -1 and repeated $\Lambda_{2}$. The other patterns $C\left(b_{1 i}\right)$ is presented in Table II. As for $F_{1}\left(b_{1 i}\right)$, it starts from 1 and ends by $2\left[\left(1-2^{i}\right) \Lambda_{1}\right]+1$ where each term is repeated $\sqrt{M_{2}}$ as shown in Table II. Similarly, the $F_{2}\left(b_{1 i}\right)$ patterns can be obtained from Table II which is repeated $\sqrt{M_{2}}$ times.

From the regularities shown in Table II, the probability that the 


\begin{tabular}{|c|c|c|c|c|c|c|c|c|c|c|c|c|c|}
\hline$C\left(b_{11}\right)$ & 1 & .. & .. & .. & 1 & 1 & .. & .. & 1 & .. & 1 & .. & 1 \\
\hline$F_{1}\left(b_{11}\right)$ & 1 & .. & .. & .. & 1 & 3 & .. & .. & 3 & .. & $\Lambda_{1}$ & .. & $\Lambda_{1}$ \\
\hline$F_{2}\left(b_{11}\right)$ & 1 & -1 & .. & .. & $\mp \Lambda_{2}$ & 1 & .. & .. & $\mp \Lambda_{2}$ & .. & 1 & .. & $\mp \Lambda_{2}$ \\
\hline$C\left(b_{12}\right)$ & 2 & .. & .. &.. & 2 & 1 & .. &.. & 1 &.. & -1 &.. & -1 \\
\hline$F_{1}\left(b_{12}\right)$ & 1 & .. & .. & .. & 1 & 3 & .. & .. & 3 & .. & $\frac{3}{2} \Lambda_{1}$ & .. & $\frac{3}{2} \Lambda_{1}$ \\
\hline$F_{2}\left(b_{12}\right)$ & 1 & -1 &.. &.. & $\mp \Lambda_{2}$ & 1 & -1 &.. & $\mp \Lambda_{2}$ &.. & 1 &.. & $\mp \Lambda_{2}$ \\
\hline$\ldots$ & $\ldots$ & & & & & & & & & & & & \\
\hline$C\left(b_{1 v_{1}}\right)$ & $\frac{1}{2} \sqrt{M_{1}}$ & .. & .. & .. & $\frac{1}{2} \sqrt{M_{1}}$ & $\frac{1}{2} \Lambda_{1}$ & & & $\frac{1}{2} \Lambda_{1}$ & .. & -1 & & -1 \\
\hline$F_{1}\left(b_{1 v_{1}}\right)$ & 1 &.. & .. &.. & 1 & 3 &.. & .. & 3 &.. & $2 \sqrt{M_{1}}-3$ & .. & $2 \sqrt{M_{1}}-3$ \\
\hline$F_{2}\left(b_{1 v_{1}}\right)$ & 1 & -1 &.. &.. & $\mp \Lambda_{2}$ & 1 & -1 & .. & $\mp \Lambda_{2}$ & .. & 1 &.. & $\mp \Lambda_{2}$ \\
\hline
\end{tabular}

TABLE II

Regular Patterns for $C\left(b_{1 i}\right), F_{1}\left(b_{1 i}\right)$ And $F_{2}\left(b_{1 i}\right)$ For the BER of the First User .

bit $b_{1 i}$ is in error can be formulated as follows:

$$
\begin{aligned}
& P_{b_{1 i}} \mid h_{1}=\frac{1}{\sqrt{M_{1}}} \sum_{j=0}^{L_{1,1}} \sum_{k=0}^{\Lambda_{2}}(-1)^{\lambda_{i, j, 1}}\left(2^{i-1}-\left\lfloor\frac{j 2^{i-1}}{\sqrt{M_{1}}}+\frac{1}{2}\right\rfloor\right) \\
& \times Q\left(\epsilon_{1}\left[(2 j+1) \sqrt{\frac{\beta_{1}}{E_{1}}}+\left(2 k-\sqrt{M_{2}}+1\right) \sqrt{\frac{\beta_{2}}{E_{2}}}\right\rfloor\right)
\end{aligned}
$$

Now, the exact closed-form for conditional BER for the first user can be obtained by averaging the BER in (13) as given by

$$
P_{U_{1}}\left|h_{1}=\frac{1}{v_{1}^{2} v_{2}} \sum_{i=1}^{v_{1}} P_{b_{1 i}}\right| h_{1} .
$$

It is worth mentioning that the results in [6] confirm that both SICD and JMuD have identical BER performance for the first user.

\section{BER EXPRESSION OF $U_{2}$}

This section considers the derivation of the BER for the $U_{2}$ in a two-user NOMA system using gray coded square $M_{n}-$ QAM modulation. The same patterns regularities approach can be followed to derive the conditional BER for the second user. The conditional BER for the second user can be expressed as follows

$$
\begin{aligned}
P_{b_{2 i}} \mid h_{\mp}=\frac{1}{\sqrt{M_{2}}} \sum_{j=0}^{L_{1,2}} \sum_{l=0}^{L_{2,1}} D_{1}(i, l, j)\left[Q\left(g_{2}^{+}\left(l 2^{\ell_{i, 1}}, 2 j+1\right)\right)\right. \\
\left.-\left(1-\delta_{l, 0}\right) Q\left(g_{2}^{-}\left(l 2^{\ell_{i, 1}}, 2 j+1\right)\right)\right]
\end{aligned}
$$

where

$$
S=l \delta_{\lfloor i-1\rfloor, 0}+\lambda_{i, j, 2}\left(1-\delta_{\lfloor i-1\rfloor, 0}\right)
$$

and

$$
\begin{array}{r}
D_{2}(i, l, j)=1+\left(\left\lfloor\frac{j}{v_{2}-1}\right\rfloor(i-1)-l\left\lfloor\frac{j}{v_{2}-1}+\frac{1}{2}\right\rfloor-1\right) \\
\times \ell_{i, 1} .
\end{array}
$$

Now, the exact conditional BER for the 1st user can be obtained by averaging the BER in (15) as given by

$$
P_{U_{2}}\left|h_{2}=\frac{1}{v_{2} \log _{2} \sqrt{m}} \sum_{i=1}^{v_{2}} P_{b_{2 i}}\right| h_{2} .
$$

It is worth mentioning that the results in [6] confirm that both SICD and JMuD have identical BER performance for the first user. Table illustrates the implementation of (14) and (19) on NOMA systems. Appendix I shows the exact BER formulae for several cases of interest.

\section{NumERICAL AND SimUlation RESUltS}

This section presents the analytical and Monte Carlo simulations results for the exact BER of NOMA systems. All users are assumed to be equipped with a single antenna. The randomly generated channels are ordered based on their strength, where the weakest channel is assigned to the 1 st user and the strongest channel is assigned to $n$th user. The channel gain values are selected randomly as follows, $\alpha_{1}=0.1789$ and $\alpha_{2}=0.6902$. The transmitted symbols for all users are selected uniformly from a Gray coded $M_{n}-$ QAM constellation. The total transmit power from the BS is unified for all cases, $P_{T}=1$. Moreover, the power allocation values $\beta_{n}$ are chosen to satisfy (6).

Fig. 2 shows the conditional BER for the first user for four different NOMA systems, $\left\{M_{1}=4, M_{2}=4\right\}$, $\left\{M_{1}=16, M_{2}=4\right\},\left\{M_{1}=4, M_{2}=16\right\}, \quad$ and $\left\{M_{1}=16, M_{2}=16\right\}$. For fair comparison, the power allocation $\beta_{1}=0.91$ and $\beta_{2}=0.09$ are unified for all the systems and chosen based on the power constraint for $\left\{M_{1}=16, M_{2}=16\right\}$. As can be noted from the figure, the analytical results obtained using (14) perfectly match the simulation results for all the considered values of $M_{1}, M_{2}$, and $E_{b} / N_{0}$. It can be noted that for low $E_{b} / N_{0}=\frac{1}{\sigma_{n_{1}}^{2}}$ values, the effect of AWGN becomes dominant and hence the BER is affected mainly by $M_{1}$. For example, for the cases where $M_{1}=16$, both $\left\{M_{1}=16, M_{2}=4\right\}$ and $\left\{M_{1}=16, M_{2}=16\right\}$ NOMA systems have the same BER performance over $E_{b} / N_{0}=[0,30] \mathrm{dB}$. As $E_{b} / N_{0}$ increases, the inter-user interference (IUI) affects the error performance. As $M_{2}$ increases, the BER performance degrades which is a result of the decreased Euclidean distance between the constellation 


$$
D_{1}(i, l, j)=(-1)^{S}\left(\sqrt{M_{1}} 2^{i-1}-\left\lfloor l 2^{i-2+\ell_{i, 2}}+\ell_{i, 1}\left(l \delta_{\rho_{i, j}, 0}+\sqrt{M_{1}}\left(1-\delta_{\rho_{i, j}, 0}\right) D_{2}(i, l, j)\right)+\frac{1}{2}\right\rfloor\right)
$$

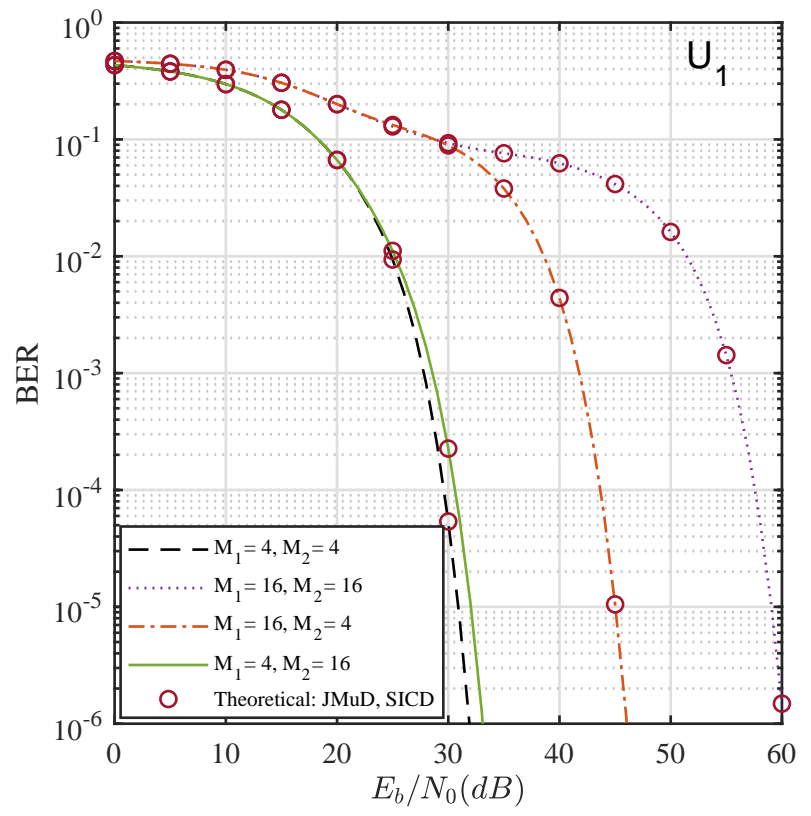

Fig. 2. Conditional BER for the first user witth different $M_{1}$ and $M_{2}$ and $\beta_{1}=0.91$.

points. For example, for the cases where $\left\{M_{1}=4, M_{2}=4\right\}$ and $\left\{M_{1}=4, M_{2}=16\right\}$, the BER for $\left\{M_{1}=4, M_{2}=4\right\}$ is lower than $\left\{M_{1}=4, M_{2}=16\right\}$ beyond $E_{b} / N_{0}=24 \mathrm{~dB}$. Moreover, it can be noted that both SICD and JMuD have identical BER performance for all considered scenarios.

Fig. 3 shows the conditional BER for the second user for four different NOMA systems, $\left\{M_{1}=4, M_{2}=4\right\}$, $\left\{M_{1}=16, M_{2}=4\right\},\left\{M_{1}=4, M_{2}=16\right\}, \quad$ and $\left\{M_{1}=16, M_{2}=16\right\}$. The power allocation value for all the system is $\beta_{1}=0.91$. It can be noticed that, the theoretical results evaluated using (19) perfectly match the simulation results for all the considered values of $M_{1}, M_{2}$, and $E_{b} / N_{0}$. It can be concluded that at low $E_{b} / N_{0}$, the effect of AWGN is controlling the error performance. For example, $\left\{M_{1}=4, M_{2}=4\right\}$ and $\left\{M_{1}=16, M_{2}=4\right\}$ have the same BER up to $5 \mathrm{~dB}$. As $E_{b} / N_{0}$ increases, the impact of IUI becomes more dominant than AWGN hence the greater $M_{1}$ is, the worse BER we obtain. This is a result of the decreased distance between the superimposed constellation points. For example, the BER for the system $\left\{M_{1}=4, M_{2}=4\right\}$ preforms better compared to the system when $\left\{M_{1}=16, M_{2}=4\right\}$. Furthermore, it can be noted that both SICD and JMuD have identical BER performance for all considered scenarios.

\section{CONCLUSION}

In this work, exact and closed-form BER expressions for twouser NOMA systems using arbitrary square $M_{n}-$ QAM per user

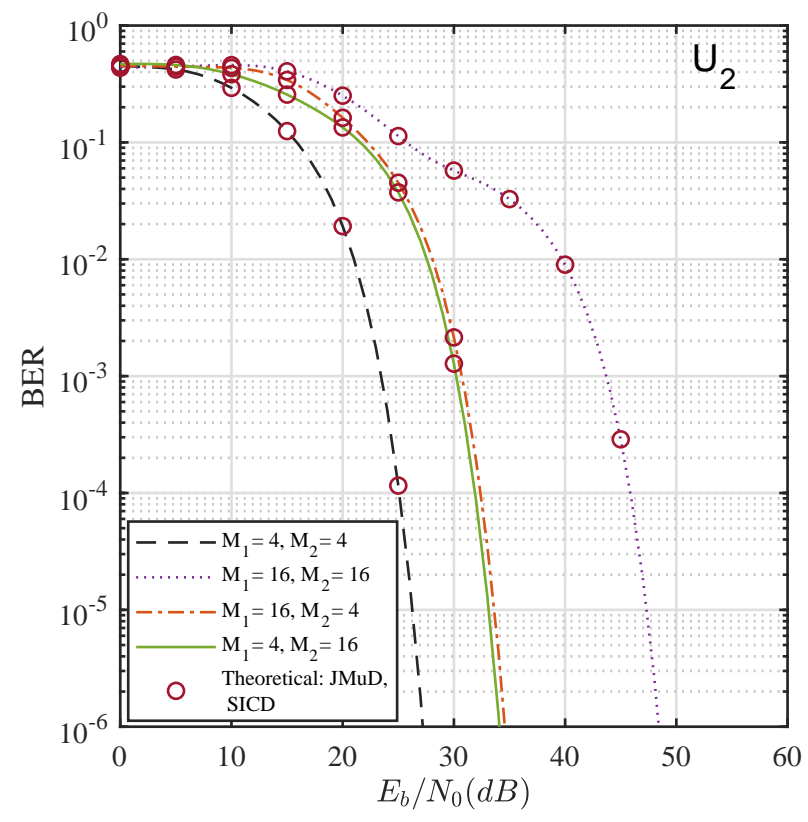

Fig. 3. Conditional BER for the second user witth different $M_{1}$ and $M_{2}$ and $\beta_{1}=0.91$.

are derived. The detection method, namely, JMuD, is considered instead of the commonly used SICD method to reduce the computational time of NOMA systems. Although the two detectors are fundamentally different, the obtained analytical and simulation results show that both detectors have identical BERs in the case of perfect channel estimates. The JMuD would be preferable for cases where the processing delay is paramount. In addition, a derived power allocation range must be satisfied in order to ensure proper NOMA implementation.

\section{APPENDIX I}

The BER for the two-user NOMA system for any $M_{1}$ and $M_{2}$ values can be obtained by direct substitution in (14) and (19). Table shows the BER for four different NOMA systems, $\left\{M_{1}=4, M_{2}=4\right\},\left\{M_{1}=4, M_{2}=16\right\}$, $\left\{M_{1}=16, M_{2}=4\right\}$, and $\left\{M_{1}=16, M_{2}=16\right\}$

A. $M_{1}=4, M_{2}=4$

$$
P_{U_{1}}=\frac{1}{2}\left[Q\left(\epsilon_{1} \varphi_{1}\right)+Q\left(\epsilon_{1} \varphi_{2}\right)\right]
$$

$$
\begin{aligned}
P_{U_{2}} & =\frac{1}{2}\left[2 Q\left(g_{2}^{+}(0,2)\right)-Q\left(g_{2}^{+}(1,1)\right)\right. \\
& \left.+Q\left(g_{2}^{+}(2,1)\right)+Q\left(g_{2}^{-}(1,1)\right)-Q\left(g_{2}^{-}(2,1)\right)\right]
\end{aligned}
$$


B. $M_{1}=4, M_{2}=16$

$$
\begin{aligned}
P_{U_{1}}=\frac{1}{4}\left[Q\left(g_{1}^{+}(1,1)\right)+Q\left(g_{1}^{-}(1,1)\right)+\right. \\
\left.Q\left(g_{1}^{+}(1,3)\right)+Q\left(g_{1}^{-}(1,3)\right)\right]
\end{aligned}
$$

$$
\begin{array}{r}
P_{U_{2}}=\frac{1}{8}\left[6 Q \left(g_{2}^{+}(0,1)+4 Q\left(g_{2}^{+}(0,3)\right)\right.\right. \\
-2 Q\left(g_{2}^{+}(0,5)\right)-Q\left(g_{2}^{+}(1,3)\right)+2 Q\left(g_{2}^{+}(2,3)\right) \\
-Q\left(g_{2}^{+}(1,1)\right)+2 Q\left(g_{2}^{+}(2,1)\right)+Q\left(g_{2}^{-}(1,1)\right) \\
-3 Q\left(g_{2}^{-}(2,1)\right)+Q\left(g_{2}^{-}(1,3)\right)-2 Q\left(g_{2}^{-}(2,3)\right) \\
\left.-\frac{1}{2} Q\left(g_{2}^{+}(2,5)\right)+Q\left(g_{2}^{-}(2,5)\right)\right]
\end{array}
$$

C. $M_{1}=16, M_{2}=4$

$$
\begin{aligned}
& P_{U_{1}}=\frac{1}{8}\left[3 Q\left(g_{1}^{+}(1,1)\right)+3 Q\left(g_{1}^{-}(1,1)\right)+2 Q\left(g_{1}^{+}\right.\right. \\
& \left.\quad+2 Q\left(g_{1}^{-}(3,1)\right)-Q\left(g_{1}^{+}(5,1)\right)-Q\left(g_{1}^{-}(5,1)\right)\right]
\end{aligned}
$$

$$
\begin{gathered}
P_{U_{2}}=\frac{1}{4}\left[4 Q\left(g_{2}^{+}(0,1)\right)-3 Q\left(g_{2}^{+}(1,1)\right)+3 Q\left(g_{2}^{+}(2,1)\right)\right. \\
-2 Q\left(g_{2}^{+}(3,1)\right)+2 Q\left(g_{2}^{+}(4,1)\right)-Q\left(g_{2}^{+}(5,1)\right) \\
+Q\left(g_{2}^{+}(6,1)\right)+3 Q\left(g_{2}^{-}(1,1)\right)-3 Q\left(g_{2}^{-}(2,1)\right) \\
+2 Q\left(g_{2}^{-}(3,1)\right)-2 Q\left(g_{2}^{-}(4,1)\right)+Q\left(g_{2}^{-}(5,1)\right) \\
-Q\left(g_{2}^{-}(6,1)\right)
\end{gathered}
$$

D. $M_{1}=16, M_{2}=16$

$$
\begin{aligned}
& P_{U_{1}}=\frac{1}{16}\left[2 Q\left(g_{1}^{+}(3,3)\right)+2 Q\left(g_{1}^{+}(3,1)\right)+2 Q\left(g_{1}^{-}(3,1)\right)\right. \\
& +2 Q\left(g_{1}^{-}(3,3)\right)+3 Q\left(g_{1}^{+}(1,3)\right)+3 Q\left(g_{1}^{+}(1,1)\right) \\
& +3 Q\left(g_{1}^{-}(1,1)\right)+3 Q\left(g_{1}^{-}(1,3)\right)-Q\left(g_{1}^{+}(5,3)\right) \\
& \left.-Q\left(g_{1}^{+}(5,1)\right)-Q\left(g_{1}^{-}(5,1)\right)-Q\left(g_{1}^{-}(5,3)\right)\right] \\
& P_{U_{2}}=\frac{1}{16}\left[12 Q\left(g_{2}^{+}(0,1)\right)-3 Q\left(g_{2}^{+}(1,1)\right)+9 Q\left(g_{2}^{+}(2,1)\right)\right. \\
& -2 Q\left(g_{2}^{+}(3,1)\right)+6 Q\left(g_{2}^{+}(4,1)\right)-Q\left(g_{2}^{+}(5,1)\right) \\
& +3 Q\left(g_{2}^{+}(6,1)\right)+4 Q\left(g_{2}^{+}(0,3)\right)-3 Q\left(g_{2}^{+}(1,3)\right) \\
& +6 Q\left(g_{2}^{+}(2,3)\right)-2 Q\left(g_{2}^{+}(3,3)\right)+4 Q\left(g_{2}^{+}(4,3)\right) \\
& -Q\left(g_{2}^{+}(5,3)\right)+2 Q\left(g_{2}^{+}(6,3)\right)-4 Q\left(g_{2}^{+}(0,5)\right) \\
& -3 Q\left(g_{2}^{+}(2,5)\right)-2 Q\left(g_{2}^{+}(4,5)\right)-Q\left(g_{2}^{+}(6,5)\right) \\
& +3 Q\left(g_{2}^{-}(1,1)\right)-9 Q\left(g_{2}^{-}(2,1)\right)+2 Q\left(g_{2}^{-}(3,1)\right) \\
& -6 Q\left(g_{2}^{-}(4,1)\right)+Q\left(g_{2}^{-}(5,1)\right)-3 Q\left(g_{2}^{-}(6,1)\right) \\
& +3 Q\left(g_{2}^{-}(1,3)\right)-6 Q\left(g_{2}^{-}(2,3)\right)+2 Q\left(g_{2}^{-}(3,3)\right) \\
& -4 Q\left(g_{2}^{-}(4,3)\right)+Q\left(g_{2}^{-}(5,3)\right)-2 Q\left(g_{2}^{-}(6,3)\right) \\
& \left.+3 Q\left(g_{2}^{-}(2,5)\right)+2 Q\left(g_{2}^{-}(4,5)\right)+2 Q\left(g_{2}^{-}(6,5)\right)\right]
\end{aligned}
$$

\section{REFERENCES}

[1] L. Dai et al., "A survey of non-orthogonal multiple access for 5G," IEEE Commun. Surv. Tut., vol. 20, no. 3, pp. 2294-2323, May 2018.

[2] M. Al-Imari et al., "Subcarrier and power allocation for LDS-OFDM system," in VTC, May 2011, pp. 1-5.

[3] F. Kara and H. Kaya, "On the error performance of cooperative-NOMA with statistical CSIT," IEEE Commun. Lett., vol. 23, no. 1, pp. 128-131, Jan. 2019.

[4] S. M. R. Islam et al., "Power-domain non-orthogonal multiple access (NOMA) in 5G systems: Potentials and challenges," IEEE Commun. Surveys Tuts, vol. 19, no. 2, pp. 721-742, Oct. 2017.

[5] J. Ding, J. Cai, and C. Yi, "An improved coalition game approach for MIMO-NOMA clustering integrating beamforming and power allocation," IEEE Trans. Veh. Technol., vol. 68, no. 2, pp. 1672-1687, Feb. 2019.

[6] T. Assaf et al., "Exact BER performance analysis for downlink NOMA systems over Nakagami- $m$ fading channels," IEEE Access, 2019.

[7] Q. He, Y. Hu, and A. Schmeink, "Closed-form symbol error rate expressions for non-orthogonal multiple access systems," IEEE Trans. Veh. Technol., vol. 68, no. 7, pp. 6775-6789, Jul. 2019.

[8] F. Kara and H. Kaya, "BER performances of downlink and uplink NOMA in the presence of SIC errors over fading channels," IET Commun., vol. 12 , no. 15 , pp. $1834-1844$, Sep. 2018.

[9] M. Zeng et al., "Capacity comparison between MIMO-NOMA and MIMO-OMA with multiple users in a cluster," IEEE J. Sel. Areas Commun., vol. 35, no. 10, pp. 2413-2424, Oct. 2017.

[10] S. K. Zaidi, S. F. Hasan, and X. Gui, "Evaluating the ergodic rate in SWIPT-aided hybrid NOMA," IEEE Commun. Lett., vol. 22, no. 9, pp. 1870-1873, Sep. 2018.

[11] L. Bariah, S. Muhaidat, and A. Al-Dweik, "Error probability analysis of non-orthogonal multiple access over nakagami- $m$ fading channels," IEEE Transactions on Communications, vol. 67, no. 2, pp. 1586-1599, 2019.

[12] B. K. Ng and C. Lam, "Joint power and modulation optimization in two-user non-orthogonal multiple access channels: A minimum error probability approach," IEEE Trans. Veh. Technol., vol. 67, no. 11, pp. $10693-10703$, Nov. 2018.

[13] C. Yan et al., "Receiver design for downlink non-orthogonal multiple access (NOMA)," in IEEE VTC-Spring), May 2015, pp. 1-6.

[14] M. Qiu et al., "A lattice-partition framework of downlink non-orthogonal multiple access without SIC," IEEE Trans. Commun., vol. 66, no. 6, pp. 2532-2546, June 2018.

[15] S. Shieh et al., "On gray labeling for downlink non-orthogonal multiple access without SIC," IEEE Commun. Lett., vol. 20, no. 9, pp. 1721-1724, Sep. 2016.

[16] F. Kara and H. Kaya, "Performance analysis of ssk-noma," IEEE Transactions on Vehicular Technology, vol. 68, no. 7, pp. 6231-6242, 2019.

[17] M. Aldababsa, C. Goztepe, G. K. Kurt, and O. Kucur, "Bit error rate for NOMA network," IEEE Communications Letters, pp. 1-1, 2020.

[18] E. M. Almohimmah and M. T. Alresheedi, "Error analysis of noma-based vlc systems with higher order modulation schemes," IEEE Access, vol. 8, pp. 2792-2803, 2020. 